\title{
Control of ion density distribution by magnetic traps for plasma electrons
}

Oleg Baranov, Maxim Romanov, Jinghua Fang, Uros Cvelbar, and Kostya (Ken) Ostrikov

Citation: Journal of Applied Physics 112, 073302 (2012); doi: 10.1063/1.4757022

View online: http://dx.doi.org/10.1063/1.4757022

View Table of Contents: http://aip.scitation.org/toc/jap/112/7

Published by the American Institute of Physics

\section{AlP | Journal of A1Pplied Physics}

Save your money for your research.

It's now FREE to publish with us no page, color or publication charges apply.
Publish your research in the

Joumal of Applied Physics

to claim your place in applied

physics history. 


\title{
Control of ion density distribution by magnetic traps for plasma electrons
}

\author{
Oleg Baranov, ${ }^{1}$ Maxim Romanov, ${ }^{1}$ Jinghua Fang, ${ }^{2,3}$ Uros Cvelbar, ${ }^{4}$ \\ and Kostya (Ken) Ostrikov 2,5 \\ ${ }^{1}$ Plasma Laboratory, National Aerospace University “KhAI," Kharkov 61070, Ukraine \\ ${ }^{2}$ Plasma Nanoscience Centre Australia (PNCA), CSIRO Materials Science and Engineering, P.O. Box 218, \\ Lindfield, New South Wales 2070, Australia \\ ${ }^{3}$ School of Physics, University of Melbourne, Parkville, VIC 3010, Australia \\ ${ }^{4}$ Jozef Stefan Institute, Jamova cesta 39, SI-1000 Ljubljana, Slovenia \\ ${ }^{5}$ The University of Sydney, Sydney, NSW 2006, Australia
}

(Received 31 May 2012; accepted 5 September 2012; published online 3 October 2012)

\begin{abstract}
The effect of a magnetic field of two magnetic coils on the ion current density distribution in the setup for low-temperature plasma deposition is investigated. The substrate of $400 \mathrm{~mm}$ diameter is placed at a distance of $325 \mathrm{~mm}$ from the plasma duct exit, with the two magnetic coils mounted symmetrically under the substrate at a distance of $140 \mathrm{~mm}$ relative to the substrate centre. A planar probe is used to measure the ion current density distribution along the plasma flux cross-sections at distances of 150, 230, and $325 \mathrm{~mm}$ from the plasma duct exit. It is shown that the magnetic field strongly affects the ion current density distribution. Transparent plastic films are used to investigate qualitatively the ion density distribution profiles and the effect of the magnetic field. A theoretical model is developed to describe the interaction of the ion fluxes with the negative space charge regions associated with the magnetic trapping of the plasma electrons. Theoretical results are compared with the experimental measurements, and a reasonable agreement is demonstrated. (C) 2012 American Institute of Physics. [http://dx.doi.org/10.1063/1.4757022]
\end{abstract}

\section{INTRODUCTION}

Plasma processing is widely used for thin film deposition, etching, modification, and doping surface layers for microelectronic, optical, biomedical, and nanotechnology applications. ${ }^{1-7}$ Ion current density is one of the key parameters which should be precisely controlled when a uniform film or nanostructured pattern need to be produced over the entire substrate surface. ${ }^{8-15}$

Up to date, vacuum arc deposition and unbalanced magnetron sputtering are the most attractive techniques to deposit thin metal films and nanostructured patterns onto the substrates. ${ }^{16,17}$ The magnetic field as a guide for the plasma from the source to the substrate is a common feature of these techniques. The application of an additional magnetic field to enhance the ion flux and affect the distribution of the ion current density over the substrate was reported. ${ }^{18-20}$ In this case, the magnetic field generated by additional electromagnetic coils placed under the substrate interacts with the field generated by the focusing and guiding coils.

Nevertheless, control of the ion density over the substrate is still a problem. Different configurations were proposed to achieve a required current distribution on the surface. Hopwood et al ${ }^{21}$ proposed a scheme with multipole confinement developed by Chen. ${ }^{22}$ Tang and $\mathrm{Chu}^{23}$ used permanent magnets and external Helmholtz type electromagnetic coils outside the discharge chamber for the inductive discharge. Wave-driven discharges are also widely used, and a large-diameter ECR plasma was produced by adjusting a multi-slot antenna. ${ }^{24}$ A technological setup with the magnetically neutral loop plasma source was also proposed. ${ }^{25}$ In this setup, a set of external electromagnets was used to control the ion flux and its space distribution. To increase the current density of metal ions from a magnetron source to the substrate, it was also proposed to weaken the inner magnet of the magnetron relative to its outer magnet, which was developed for the unbalanced magnetrons. ${ }^{26}$ Another possible configuration included a coil under the substrate in the setup with the unbalanced magnetron. ${ }^{27}$ A coil placed in front of the target was also used to improve performance of a high power impulse magnetron sputtering (HIPIMS) setup. ${ }^{28}$ For the vacuum arc deposition setups, Anders ${ }^{29}$ described a twist filter, which can be used to direct the ions toward the particular area of the substrate. Bilek and co-authors ${ }^{30}$ investigated a current-driven plasma homogenizer above the deposition plane for the purpose.

One of the promising approaches for controlling the ion current density is the use of the "bottle" and "cusp" magnetic field configurations. ${ }^{20}$ By varying the combinations of the "bottle" and "cusp" magnetic field configurations, different ion current density distributions over the substrate were obtained. $^{31}$ The strength of the resulting magnetic field allowed trapping the plasma electrons with the ions remaining un-magnetized. This leads to a self-consistent electric field that facilitates effective transport of the plasma from the cathode of the vacuum arc source. ${ }^{29,32}$

However, the above-mentioned systems do not ensure the required controllability level of the ion current distribution over the large substrates, especially in the high-current setups that combine vacuum arc and magnetron-like plasma sources. Much more sophisticated design and magnetic field configurations are required to satisfy the present-day requirements. Besides, a much better understanding of the underlying physics is required to design highly-efficient, vacuum 
arc-based equipment suitable for the implementation of the present-day nanotechnology processes. In this paper, we investigate the ion current distribution over the substrate surface immersed into a complex magnetic field formed by (i) magnetic coils installed under the substrate; (ii) magnetic coil of the plasma guide. We demonstrate that in such configuration, several efficient magnetic traps for plasma electrons are formed in the region between the plasma source and the substrate. We also study the plasma parameters, distribution of ion fluxes on the surface, and demonstrate that in the configuration proposed, an effective control of the current distribution is possible. Our conclusions are supported by the numerical modeling. The proposed configuration can be used to enable effective control of the ion fluxes extracted from vacuum arc guns and unbalanced magnetrons that produce metal ions, and from the wave-driven (e.g., ECR or helicon) plasma sources that produce gaseous ions. ${ }^{33-35}$

\section{EXPERIMENTAL SETUP AND PROCEDURE}

The experimental setup is shown schematically in Fig. 1. It consists of a vacuum arc plasma source, two additional electromagnetic coils with ferromagnetic cores (Coil 1 and Coil 2), and a planar probe for measuring the radial distribution of an axial component of the ion flux (not shown). The diameter of the front surface of the truncated cone-shaped titanium cathode was $50 \mathrm{~mm}$. The anode had a $210 \mathrm{~mm}$ inner diameter and a length of $200 \mathrm{~mm}$. The focusing magnetic field of $0.03 \mathrm{~T}$ at the centre of the focusing coil was used to retain the cathode spots on the front cathode surface. The guiding magnetic field $B_{g}$ of $0.02 \mathrm{~T}$ at the centre of the guiding coil guided the plasma beam towards the substrate. A guiding coil was mounted on the anode, so the anode was used as a plasma duct. The plasma source was mounted on a flange of a $500 \mathrm{~mm}$ diameter, $500 \mathrm{~mm}$ long cylindrical vacuum chamber. When operating the plasma source, the dc arc current $I_{a}=110 \mathrm{~A}$ was applied between the cathode and the grounded anode.

A disk-shaped substrate made of nonmagnetic stainless steel was placed coaxially with the plasma duct, at a distance of $325 \mathrm{~mm}$ from the plasma duct exit. The substrate diameter and thickness were $400 \mathrm{~mm}$ and $8 \mathrm{~mm}$, respectively. Two additional coils with diameters of $100 \mathrm{~mm}$ and heights of

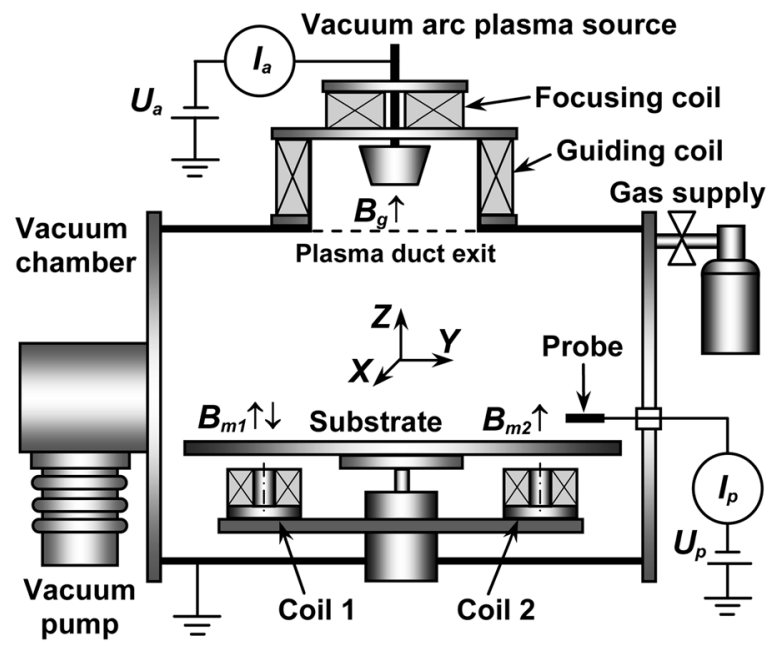

FIG. 1. Experimental setup.
$80 \mathrm{~mm}$ (2000 turns on a core of $30 \mathrm{~mm}$ diameter) were mounted symmetrically on a holder under the substrate at a distance of $140 \mathrm{~mm}$ from the substrate centre. The measured dependence of the magnetic field generated by the additional coil at the substrate surface showed an almost linear dependence on the coil current, with the magnetic fields of $0.05 \mathrm{~T}$ and $0.19 \mathrm{~T}$ for coil currents of $1.0 \mathrm{~A}$ and $4.0 \mathrm{~A}$, respectively. During the process, the substrate was under a negative potential relative to the grounded vacuum chamber walls. An automated gas-handling system maintained a nitrogen pressure of $0.01 \mathrm{~Pa}$.

A planar Langmuir probe was used to map the ion current density for three cross-sections of the plasma flux at a distance $d(150,230$, and $325 \mathrm{~mm})$ from the plasma duct exit (along $z$ axis on Fig. 1), as shown in Fig. 2(a). The probe was positioned at the points $\left(r_{p}, \varphi_{p}\right)$ with $r_{p}=35,70,105,150$, $200 \mathrm{~mm}$, and $\varphi_{p}=n \pi / 4(n=0,1 \ldots 7)$ for each cross-section. The probe was a $10 \times 10 \times 0.5 \mathrm{~mm}^{3}$ current-collecting plate made of polished nonmagnetic stainless steel with a hightemperature insulator on one side; the probe construction is shown in Fig. 2(b). Single probe was used to obtain the current density and the map of density distribution to prevent the influence of the multi-probe setup on the magnetic confinement of the plasma electrons through the possible nearwall conductivity mechanism. ${ }^{36}$ The probe was connected to the power supply via a separate ammeter. When the vacuum arc plasma source was switched on, the voltage drop between the probe and anode was $200 \mathrm{~V}$, and the saturated ion current was collected. ${ }^{37,38}$ The duration of each experimental run was $2 \mathrm{~s}$. In addition, to obtain the plasma density profiles on the substrate, the deposition profiles were mapped by placing planar deposition probe $\left(\mathrm{a} 40 \times 40 \mathrm{~cm}^{2}\right.$ piece of a transparent plastic film) on the substrate surface. The time of the film exposure to the plasma was $5 \mathrm{~s}$.
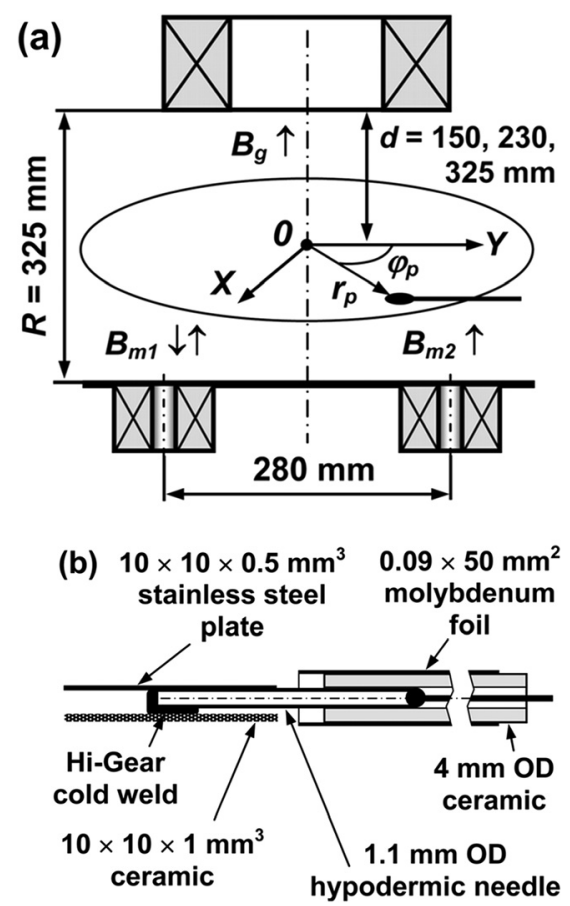

FIG. 2. Schematic of the ion current density measurements (a); schema of Langmuir planar electrical probe (b). 


\section{EXPERIMENTAL RESULTS}

The configurations, when both the additional coils generate magnetic fields aligned with the field from the guiding and focusing coils, are denoted as $B_{m 1} \uparrow B_{g} \uparrow B_{m 2} \uparrow$. The configurations are denoted as $B_{m 1} \downarrow B_{g} \uparrow B_{m 2} \uparrow$ when the magnetic field generated by one additional coil (Coil 1) is directed opposite to the magnetic field of the guiding and focusing coils, while the magnetic field generated by another coil (Coil 2 ) is aligned with the magnetic field of the guiding and focusing coils. Photographs of the plasma discharge for different powers supplied to the additional coils during the operation of the vacuum arc plasma source are shown in Fig. 3.

Figure 4 shows the ion current density distributions measured by the planar probe. These plots show the results of an approximation of the experimental data points by their average values over 10 measurements. A uniform current distribution over the probe plate was assumed. The continuous distribution in Fig. 4 was obtained by a sum of functions $\sum a_{i} \exp \left[-\left(y-y_{i}\right)^{2} / b_{i}^{2}-\left(x-x_{i}\right)^{2} / c_{i}^{2}\right]$ to fit the measured values with the error not exceeding $10 \%$ at the points of the probe location. In the $x-y$ plane shown in Fig. 1, the substrate center is located at point $x=0$ and $y=0$, while the additional coils are located under the substrate at $y=$ $\pm 140 \mathrm{~mm}, x=0$. The plots corresponding to the probe location at distances of $150 \mathrm{~mm}, 230 \mathrm{~mm}$, and $325 \mathrm{~mm}$ from the plasma duct exit along $z$-axis are shown in Figs. 4(a)-4(d), 4(e) $-4(\mathrm{~h})$, and 4(k)-4(n), respectively.

The semi-transparent deposit (titanium film) on the planar deposition probe (transparent plastic film) was optically scanned, and the images were processed by the method developed by Bilek and Brown. ${ }^{39}$ The unprocessed images of the plasma deposition are shown in Figs. 5(a), 5(c), 5(e), and $5(\mathrm{~g})$. After four applications of the triangular grey scale mapping function, contour lines of the optical density were generated where the black and white lines show linearly varying contours of the optical density, correlating logarithmically with the film thickness. Figs. 5(b), 5(d), 5(f), and 5(h) correspond to the deposits shown in Figs. 5(a), 5(c), $5(\mathrm{e})$, and $5(\mathrm{~g})$, respectively, after the application of the mapping function.

The measurements revealed the following effect of the additional coils on the ion flux configuration. When the additional coils are not powered, the uniform expansion of the ion flow extracted from the plasma source is observed (Figs. 3(a), 4(a), 4(e), 4(k), and 5(a), and (b)).

When the right coil (coil 2, $B_{g} \uparrow B_{m 2} \uparrow$, Fig. 1) is powered only, the plasma flux is contracted. The most interesting feature of this configuration is that the ion jet was extracted from the mainstream and directed toward the powered coil (Figs. 3(b), 4(b), 4(f), 4(l), 5(c), and 5(d)). The photograph in Fig. 3(b) demonstrates the effect of the bottle configuration of the magnetic field between the plasma duct exit and coil 2. The maximum ion current density increased by a factor of 1.4 from $240 \mathrm{Am}^{-2}$ (Fig. 4(a)) to $340 \mathrm{Am}^{-2}$ (Fig. 4(b)) for the distance of $150 \mathrm{~mm}$, with the distribution maximum located at $y=0$. For the distance of $230 \mathrm{~mm}$, the current increased in 1.3 times from $92 \mathrm{Am}^{-2}$ (Fig. 4(e)) to $117 \mathrm{Am}^{-2}$ (Fig. 4(f)), with the maximum located at $y \approx 0 \mathrm{~mm}$. And finally, for the distance of $325 \mathrm{~mm}$, the maximum ion current density increased by in 1.5 times from $50 \mathrm{Am}^{-2}$ (Fig. 4(k)) to 75 $\mathrm{Am}^{-2}$ (Fig. 4(1)), with the maximum of the extracted ion jet distribution (of about $75 \mathrm{Am}^{-2}$ ) located at $y \approx 120 \mathrm{~mm}$. It should be noted that the arc current decreased in this configuration from 110 to $100 \mathrm{~A}$ for the coil current of $I_{m 2}=4 \mathrm{~A}$.

Two ion jets are extracted from the mainstream and directed toward the simultaneously powered additional coils (configuration $B_{m 1} \uparrow B_{g} \uparrow B_{m 2} \uparrow$, Figs. 3(c), 4(c), 4(g), 4(m), $5(\mathrm{e})$, and $5(\mathrm{f}))$. In this configuration, the interaction of the
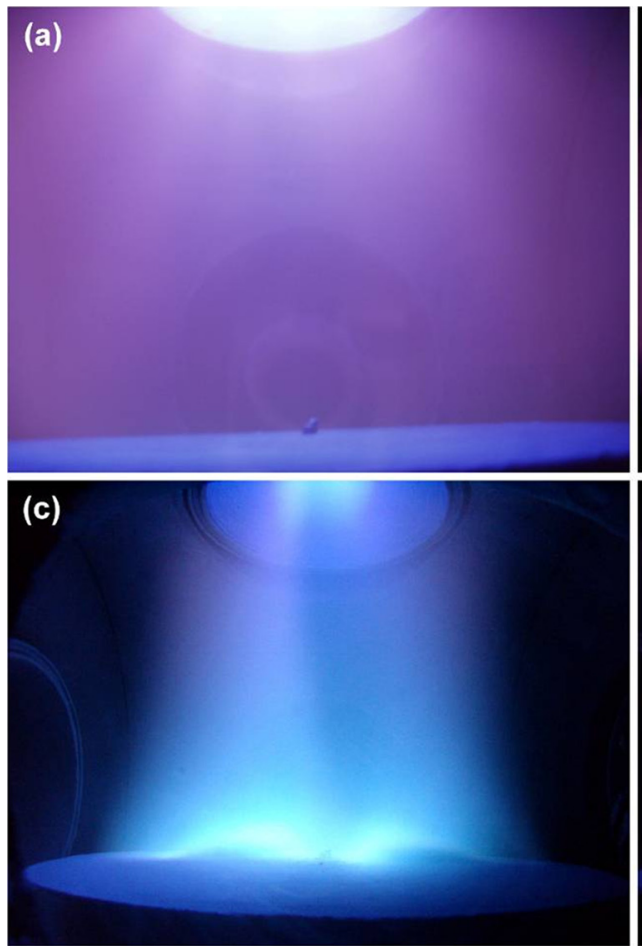

(b)
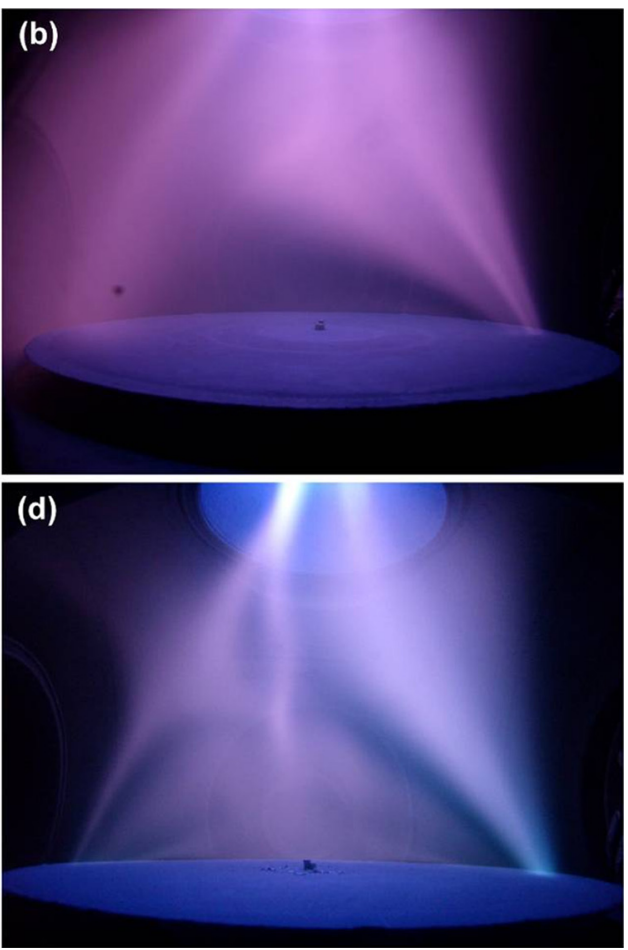

FIG. 3. Photo of the plasma structure for different configurations of the resulting magnetic field, with the vacuum arc plasma source operating: (a) $-B_{g} \uparrow$; (b) - $B_{g} \uparrow B_{m 2} \uparrow, I_{m 2}=4.0 \mathrm{~A}$; (c) $-B_{m 1} \uparrow B_{g} \uparrow$ $B_{m 2} \uparrow, \quad I_{m 1}=I_{m 2}=4.0 \mathrm{~A}$; (d) $-B_{m 1} \downarrow B_{g} \uparrow$ $B_{m 2} \uparrow, I_{m 1}=I_{m 2}=4.0 \mathrm{~A}$. 

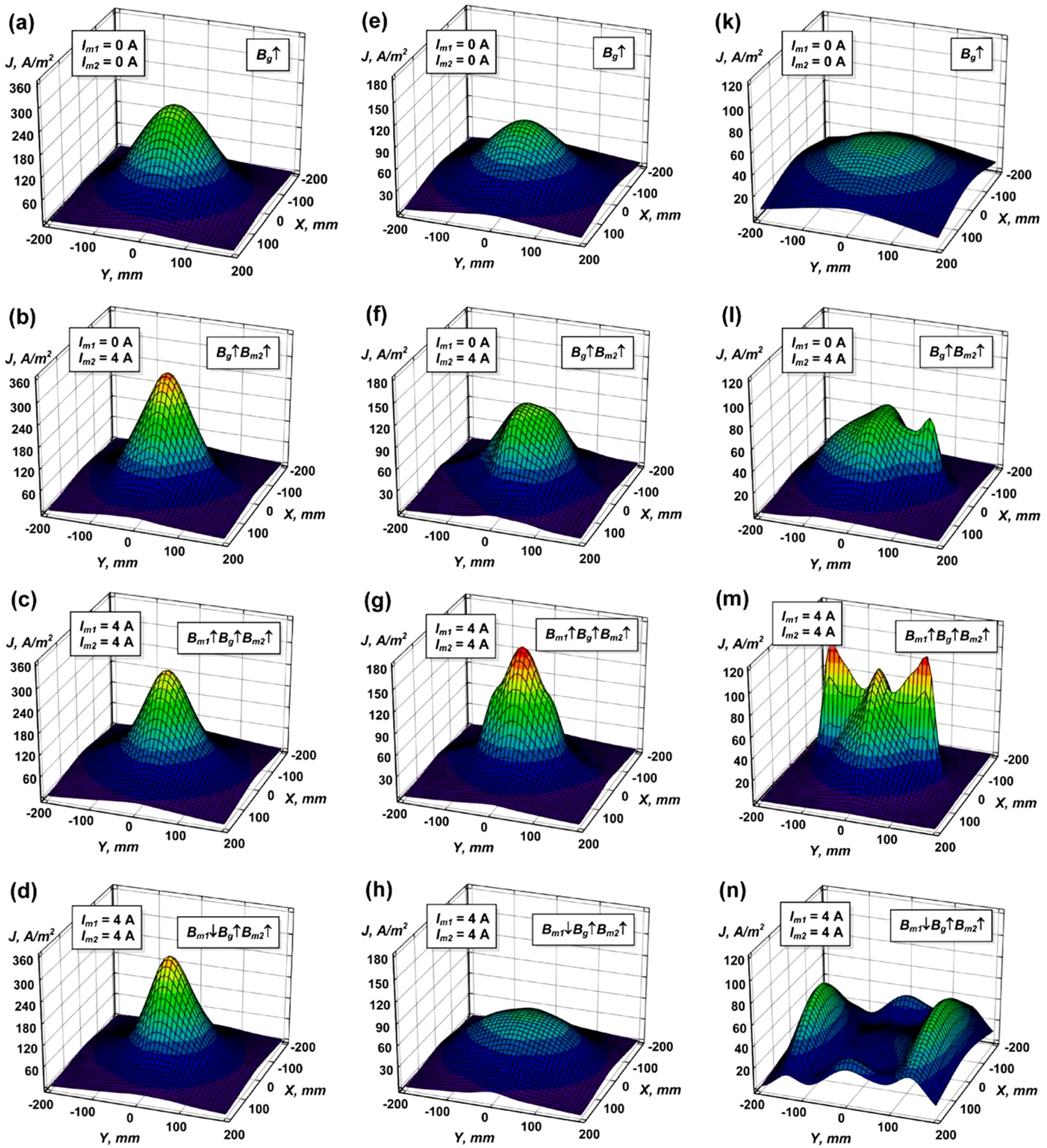

FIG. 4. Ion current density distribution as a function of coordinate along the substrate surface for the probe located at a distance of $150 \mathrm{~mm}$ (a)-(d), $230 \mathrm{~mm}$ (e)-(h), and $325 \mathrm{~mm}(\mathrm{k})-(\mathrm{n})$ from the plasma duct exit. Configuration of the resulting magnetic field: (a), (e), (k) $-B_{g} \uparrow ;$ (b), (f), (l) $-B_{g} \uparrow B_{m 2} \uparrow, I_{m 2}=4.0 \mathrm{~A}$; (c), (g), (m) $-B_{m 1} \uparrow B_{g} \uparrow B_{m 2} \uparrow, I_{m 1}=I_{m 2}=4.0 \mathrm{~A}$; and (d), (h), (n) $-B_{m 1} \downarrow B_{g} \uparrow B_{m 2} \uparrow, I_{m 1}=I_{m 2}=4.0 \mathrm{~A}$.

magnetic cusp formed between the additional coils with the two magnetic "bottles" between the coils and the plasma duct exit determines the ion flux distribution. The cusp is located right above the substrate centre. Thus, the plasma propagates from the arc source in three directions: two magnetic mirrors above the coil cores and the magnetic cusp between them. In this configuration, the maximum ion current density increased in 1.3 times from $240 \mathrm{Am}^{-2}$ (Fig. 4(a)) to 320 $\mathrm{Am}^{-2}$ (Fig. 4(c)) for the distance of $150 \mathrm{~mm}$ (maximum located at $y=0$ ), and in 2 times from $92 \mathrm{Am}^{-2}$ (Fig. 4(e)) to $183 \mathrm{Am}^{-2}$ (Fig. 4(g)) for the distance of $230 \mathrm{~mm}$, with the maximum located at $y=0$. Interestingly, the three maxima of the ion current distribution located at $x=0, y=0$ and $y= \pm 110 \mathrm{~mm}$ were found for the distance of $325 \mathrm{~mm}$. The ion 

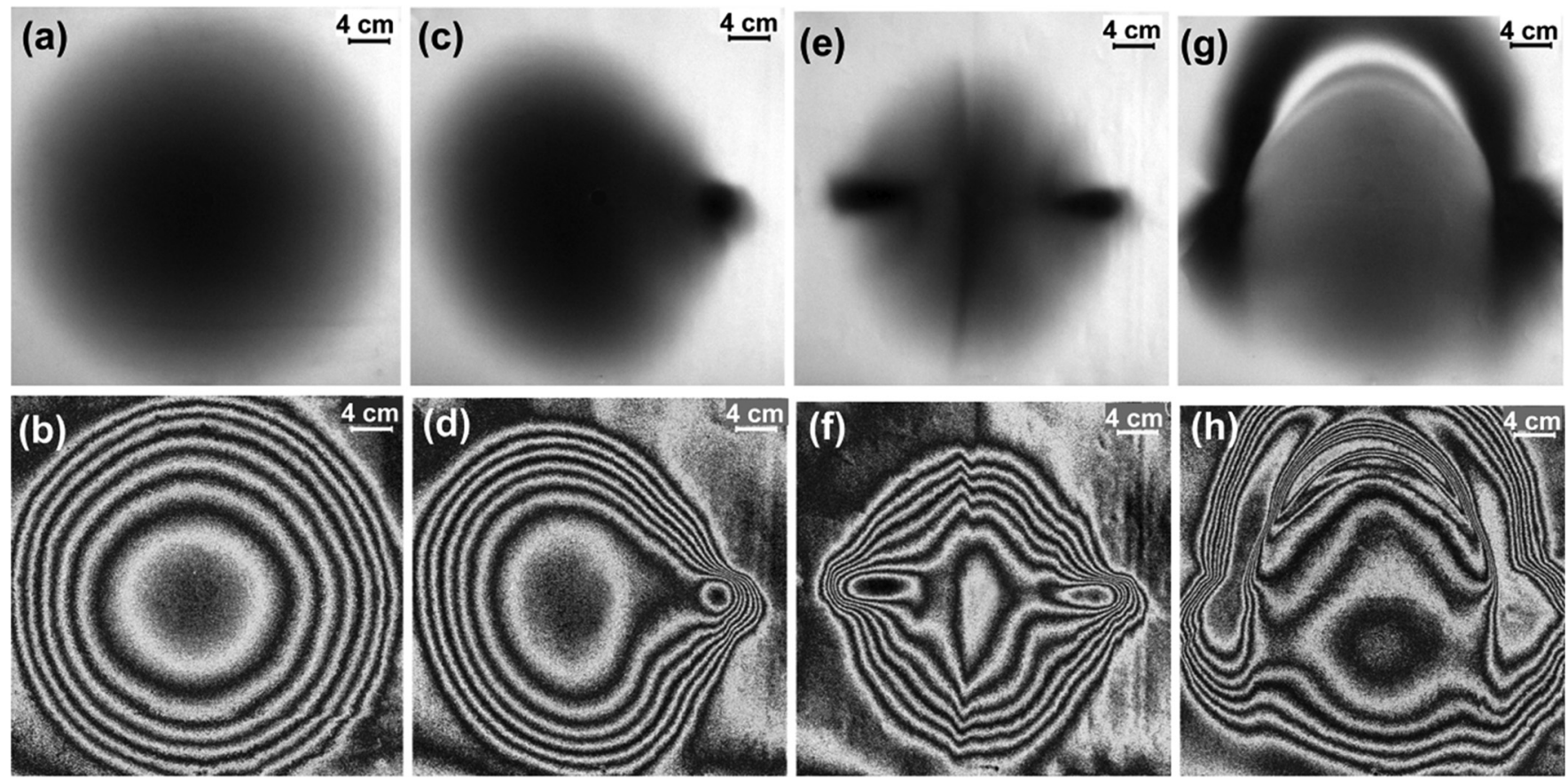

FIG. 5. Images obtained by optical scanning of the transparent planar deposition probe, before (a), (c), (e), (g) and after (b), (d), (f), (h) the application of mapping function, for different configurations of resulting magnetic field: (a), (b) $-B_{g} \uparrow$; (c), (d) $-B_{g} \uparrow B_{m 2} \uparrow, I_{m 2}=4.0 \mathrm{~A}$; (e), (f) $-B_{m 1} \uparrow B_{g} \uparrow B_{m 2} \uparrow, I_{m 1}=I_{m 2}=4.0 \mathrm{~A}$; (g), (h) $-B_{m 1} \downarrow B_{g} \uparrow B_{m 2} \uparrow, I_{m 1}=I_{m 2}=4.0 \mathrm{~A}$.

current density increased by a factor of 2 from $50 \mathrm{Am}^{-2}$ (Fig. 4(k)) to $100 \mathrm{Am}^{-2}$ for the central maximum $(y=0)$, and in almost 3 times from $40 \mathrm{Am}^{-2}$ to $117 \mathrm{Am}^{-2}$ for the side maxima $(y= \pm 110 \mathrm{~mm})$, see Figs. $4(\mathrm{k})$ and $4(\mathrm{~m})$. The arc current decreased from 110 to $95 \mathrm{~A}$, when the both coils were powered simultaneously by the current of $4.0 \mathrm{~A}$.

When the directions of magnetic fields in the coils were opposite ( $B_{m 1} \downarrow B_{g} \uparrow B_{m 2} \uparrow$ configuration), the substrate centre is screened from penetration of the ion flux extracted from the arc source (see Figs. 3(d), 4(d), 4(h), 4(n), 5(g), and 5(h)). The magnetic bottle formed between the additional coils near the substrate surface is the most probable reason for this screening. In this case, the ion flux propagates from the arc source to the substrate edge, focusing toward the magnetic mirrors above the additional coil cores. An interesting feature of this configuration is a clearly shaped space between the dark deposit at the substrate edge and a thin (semi-transparent) deposit in the central area of the substrate (Fig. 5(g)). In this configuration, the maximum ion current density increased in 1.25 times from 240 $\mathrm{Am}^{-2}$ (Fig. 4(a)) to $300 \mathrm{Am}^{-2}$ (Fig. 4(d)) for the distance of $150 \mathrm{~mm}$, with the maximum located at $y=0$, and by 0.8 from $92 \mathrm{Am}^{-2}$ (Fig. 4(e)) to $75 \mathrm{Am}^{-2}$ (Fig. 4(h)) for the distance of $230 \mathrm{~mm}$, with the maximum located at $y=10$ $\mathrm{mm}$. The ion current density decreased significantly at the substrate centre, as compared with the configuration without additional magnetic fields (by a factor of 2 from 50 to $25 \mathrm{Am}^{-2}$ ) for the distance of $325 \mathrm{~mm}$. Local maximums are located at $x=0$ and $y= \pm 140 \mathrm{~mm}$, where the ion current density increased by a factor of 2 and reached $67 \mathrm{Am}^{-2}$, as well as at $x= \pm 180 \mathrm{~mm}$ and $y=0$, where the ion current density increased by a factor of 1.5 and reached $37 \mathrm{Am}^{-2}$. The arc current was did not change for this configuration.

\section{MODEL}

Different models were developed to describe motion of plasma ions in the guiding magnetic field of vacuum arc deposition setups. Among them, the kinetic models based on Vlasov equilibrium equation, hydrodynamic and drift models, and plasma optics models are used. ${ }^{40-46}$ Most of the models require complicated numerical calculations, and thus many simplifying assumptions are used in the calculations. Also, the plasma optics models are widely used because of their relative simplicity and utility to analyze the ion motion through the plasma duct. ${ }^{46}$

A theoretical plasma optic model was developed to describe the effect of the magnetic field configuration on the ion motion. The model is based on the assumption of the formation of negative space charge regions in the plasma affected by the magnetic field of various configurations. The bottle or cusped configuration results in the formation of the magnetic traps for the plasma electrons. ${ }^{31,47}$ Within the trap, the electrons are confined by the magnetic field, while the ions are not. Hence, the electron is rather higher than the ion density and the region gains the negative electric space charge. Ions that exit the plasma duct exit are affected by the negative space charge generated in the magnetic trap region.

In our setup, a very complex magnetic field of the "bottle," "cusp," or hybrid configuration was formed, depending on the mode of operation (orientation of the magnetic field of the specific coils). To model the plasma/ion fluxes in various configurations, the formation of the magnetic traps of the specific configurations should be considered.

The electrons drift relatively slowly across the magnetic field. In a static equilibrium, the sum of the kinetic and magnetic field pressures $p+B^{2} / 2 \mu_{0}=$ const is a constant, ${ }^{22}$ 
where $B$ is the magnetic field, $\mu_{0}$ is the vacuum permeability, and $p$ is the kinetic pressure of electrons.

For the bottle or cusped configurations of the magnetic trap, more electrons are trapped near midplane of the magnetic trap then near the ends because of the mirror ratio. At the same time, the ions that exit the plasma source with kinetic energy $E_{i o n o}$ pass the magnetic trap without any significant changes in their motion. ${ }^{48,49}$ However, the ions are affected by the negative space developed in the magnetic trap. The magnetic fields were calculated, and the regions of the magnetic traps were detected. A schematic in Fig. 6(a) explains the effect of the space charge on the ion motion, where the magnetic traps layout for configuration $B_{m 1} \uparrow B_{g} \uparrow B_{m 2} \uparrow$ is shown schematically as an example.

A possibility of ion interaction with the space charge region is determined by the solution of the equation describing the intersection of the ion path $y_{k b}(z)$ with the projection of the space charge region onto the plane of the ion path $\left(z-z_{i}\right)^{2}+\left(y_{k b}(z)-y_{i}\right)^{2}=R_{\text {trap }}^{2}$, where $R_{\text {trap }}$ is the region radius, and $\left(z_{i}, y_{i}\right)$ are the coordinates of the region centre. ${ }^{50}$ When an ion intersects the border of the space charge region, it gets into a potential well. In this case, the potential energy of the interaction can be described as follows. The negative space charge is assumed to be distributed uniformly throughout the volume of the magnetic trap region. The charge density is a product $K_{q n} n_{0}$, where $K_{q n}=\left(n_{e}-n_{0}\right) / n_{0}, n_{e}-n_{0}$ is the excess of the negative charges, and $n_{0}$ is the ion density. The charge contained within the sphere of radius $r$ is $Q(r)$ $=-(4 / 3) \pi e r^{3} K_{q n} n_{0}$, and the electric field generated at $r \leq R_{\text {trap }}$ is $E(r)=-(4 / 3) \pi e K_{q n} n_{0} K_{u} r$, where $K_{u}$ is a constant. ${ }^{51}$ Thus, the potential energy of the moving positive charge $U(r)$ in the negative space charge region at $r \leq R_{\text {trap }}$ is $U(r)=\alpha_{u}\left(r^{2}-R_{\text {trap }}^{2}\right)$, where $\alpha_{u}=(2 / 3) \pi e^{2} K_{u} K_{q n} n_{0}$. It can be seen that the obtained dependence of the potential on the space coordinate is similar to the form used by Aksenov and corresponds to the result derived by Davidson. ${ }^{40,46}$

Taking into account the ion angular momentum relative to the electric field centre, the effective potential energy of the ion is: ${ }^{50}$

$$
U_{e f}(r)=\alpha_{u}\left(r^{2}-R_{\text {trap }}^{2}\right)+\frac{M_{t}^{2}}{2 M r^{2}}, \quad r \leq R_{\text {trap }} ;
$$

and $U_{e f}(r)=0$ when $r>R_{\text {trap }}$. The profile of $U_{e f}(r)$ is shown in Fig. 6(b).

Besides, the following important assumptions were adopted in our model: (1) the ion kinetic energy is above zero at the border of the potential well, and (2) ion collisions are negligible in the pressure range considered. ${ }^{19,20}$ Thus, the ion path is a straight line between the potential wells. The ion path before entering $\left(y_{k b}\right)$, inside $\left(y_{t}\right)$, and after leaving the well are sketched in Fig. 6(c). The ion motion in the potential well is a plane motion of a particle in a central field, which is described in the polar coordinates $(r, \varphi)$ with the origin in the center of the field (center of the potential well, in our case). ${ }^{50}$ To describe the ion motion in the negative space charge region, the known equations should be solved ${ }^{50}$

$$
\begin{gathered}
t=\int \frac{d r}{\sqrt{\frac{2}{M}\left[E_{t}-U_{e f}(r)\right]}}+\text { const, } \\
\phi=\int \frac{M_{t} d r}{r^{2} \sqrt{2 M\left(E_{t}-U_{e f}(r)\right)}}+\text { const },
\end{gathered}
$$

where $2 E_{t}=M\left(V_{i 0} \cos \psi_{0}\right)^{2}+M_{t}^{2} / M R_{\text {trap }}^{2}=2 E_{\text {ion } 0}$ is a total ion energy, $M_{t}=M \rho_{0} V_{i 0}, \quad \psi_{0}=\arcsin \left(\rho_{0} / R_{\text {trap }}\right), \quad V_{i 0}=$
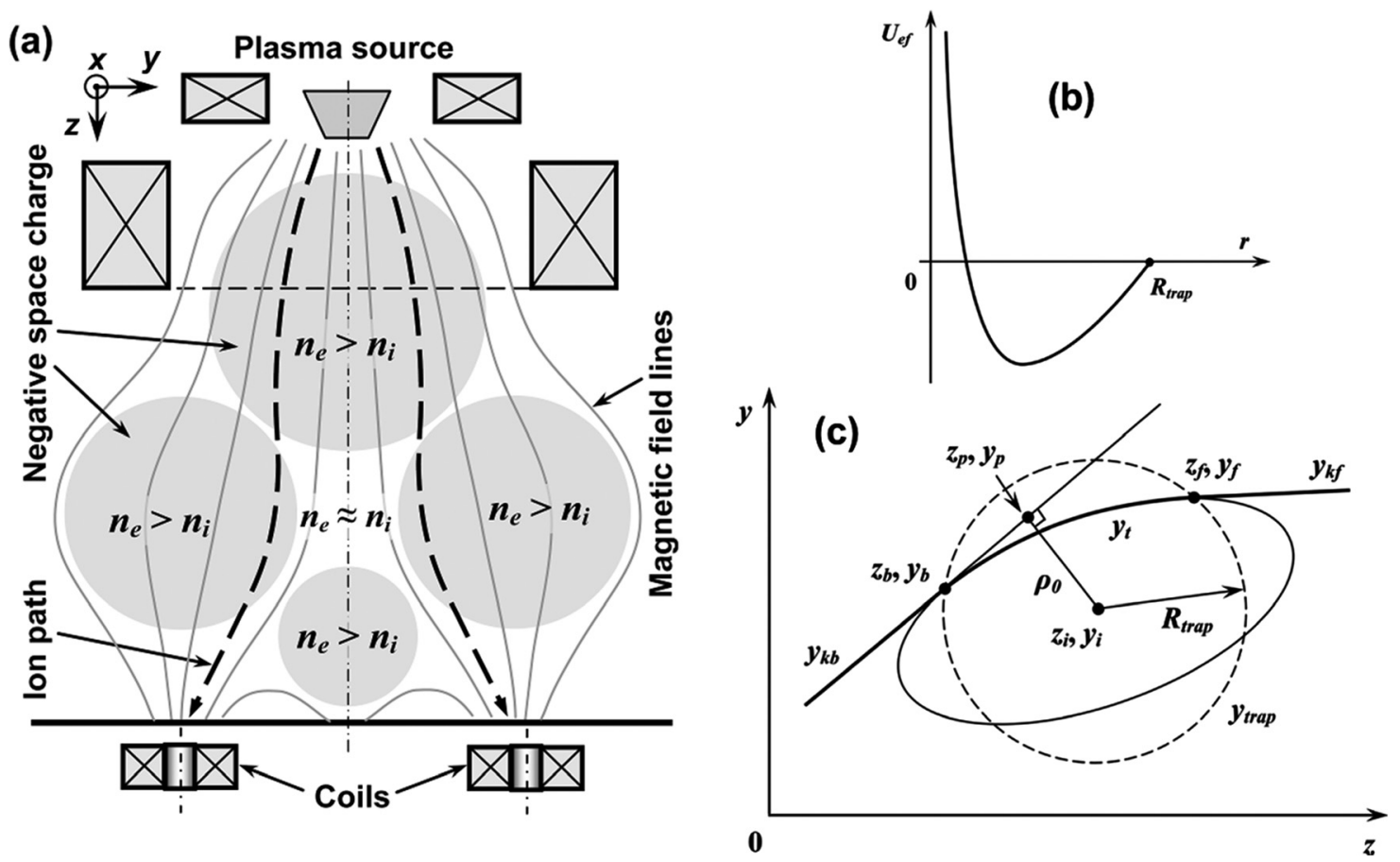

FIG. 6. Schematic of the ion fluxes in the presence of negative space charge regions (a); profile of the ion potential energy in the negative space charge region (b); ion path within negative space charge region (c). 
$\sqrt{2 e E_{\text {ion } 0} / M}$ is the ion velocity, $E_{\text {ion } 0}$ are the ion kinetic energy at the border of the potential well, $\rho_{O}$ is the impact parameter (Fig. 6(c)), and $M$ is the ion mass. When calculating the impact parameter, a distance from the centre of the space charge region to a tangent line of the ion path at the point of the intersection with the border of the space charge region should be determined as shown in Fig. 6(c).

The tangent line at the point $\left(x_{b}, y_{b}\right)$ of the intersection with the border $y_{\text {trap }}$ of the space charge region can be derived from the relation $y_{k b}=y_{b}+y_{k b}^{\prime}\left(x_{b}\right)\left(x-x_{b}\right)$. The impact parameter $\rho_{0}$ can be found as the shortest distance from the point $\left(x_{b}, y_{b}\right)$ to the tangent line $\quad l_{d k}=\sqrt{\left(x-x_{i}\right)^{2}+\left(y_{k b}-y_{i}\right)^{2}}, \quad$ i.e. $\quad \rho_{0}=$ $\sqrt{\left(y_{i}-y_{b}-y_{k b}^{\prime}\left(x_{b}\right)\left(x_{i}-x_{b}\right)\right)^{2} /\left(1+y_{k b}^{\prime}{ }^{2}\left(x_{b}\right)\right)}$.

Coordinates of the $\left(z_{p}, y_{p}\right)$ point are determined by the expressions (Fig. 6(c))

$$
\begin{gathered}
x_{p}=\sqrt{\frac{\left(x_{i}-x_{b}\right)^{2}+\left(y_{i}-y_{b}\right)^{2}-\rho_{0}^{2}}{1+y_{k b}^{\prime}\left(x_{b}\right)}}+x_{b}, \\
y_{p}=y_{b}+y_{k b}^{\prime}\left(x_{b}\right)\left(x_{p}-x_{b}\right) .
\end{gathered}
$$

Equation (2) determines the distance $r$ of an ion from the region centre as an implicit function of time $t$, and Eq. (3) determines the relation between radius $r$ and angle $\varphi$.
The equation of the path can be found by substitution of Eqs. (1) into (3) and integration of the result

$$
\varphi=\frac{1}{2} \arcsin \left(\left(\frac{E_{e f}}{\alpha_{u}} r^{2}-\frac{M_{t}^{2}}{M \alpha_{u}}\right) / r^{2} \sqrt{\frac{E_{e f}^{2}}{\alpha_{u}^{2}}-\frac{2 M_{t}^{2}}{M \alpha_{u}}}\right)+\text { const },
$$

where $E_{e f}=E_{t}+\alpha_{u} R_{\text {trap }}^{2}$. Assuming $a_{s}=\sqrt{E_{e f} / 2 \alpha_{u}}$ and $e_{s}=\sqrt{1-2 \alpha_{u} M_{t}^{2} / M E_{e f}^{2}}$, equation of the path can be written as

$$
r=a_{s} \sqrt{1-e_{s}^{2}}\left(1-e_{s} \sin 2\left(\varphi+\frac{\pi}{4}-\psi\right)\right)^{-1 / 2},
$$

where $\psi$ is the angle determined by origin.

In the Cartesian coordinates $z-y$ (Figs. 6(a) and 6(c)), the equation of the path can be obtained from Eq. (7) by substituting $z=r \cos \varphi, y=r \sin \varphi$, and $r^{2}=z^{2}+y^{2}$ :

$$
\frac{z^{2}\left(1-e_{s} \cos 2 \psi\right)}{\left(1-e_{s}^{2}\right) a_{s}^{2}}+\frac{y^{2}\left(1+e_{s} \cos 2 \psi\right)}{\left(1-e_{s}^{2}\right) a_{s}^{2}}-\frac{2 y z e_{s} \sin 2 \psi}{\left(1-e_{s}^{2}\right) a_{s}^{2}}=1 .
$$

Finally, the equation the ion path in the space charge region with centre point $\left(z_{i}, y_{i}\right)$ can be obtained

$$
y_{t}=\frac{ \pm\left(z-z_{i}\right) e_{s} \sin 2 \psi \mp \sqrt{\left(z-z_{i}\right)^{2}\left(e_{s}^{2}-1\right)+a_{s}^{2}\left(1-e_{s}^{2}\right)\left(1+e_{s} \cos 2 \psi\right)}}{1+e_{s} \cos 2 \psi}+y_{i},
$$

where the upper signs are used for $y_{p}<y_{i}$, and the lower signs - for $y_{p}>y_{i}$.

Rotation angle of the path is determined by the requirement that the equation of the tangent line to the path $y_{t}$ should describe the ion path equation in the point of intersection with boundary $y_{\text {trap }}$ of the space charge region (Fig. 6(c)). A slope of the tangent line to the path $y_{t}$ is

$$
y_{t}^{\prime}= \pm \frac{e_{s} \sin 2 \psi}{1+e_{s} \cos 2 \psi} \mp \frac{\left(z-z_{i}\right)\left(e_{s}^{2}-1\right)}{\sqrt{\left(z-z_{i}\right)^{2}\left(e_{s}^{2}-1\right)+a_{s}^{2}\left(1-e_{s}^{2}\right)\left(1+e_{s} \cos 2 \psi\right)}\left(1+e_{s} \cos 2 \psi\right)} .
$$

At $\psi=0$ the path equation is $y_{t 0}=$
$\frac{\mp \sqrt{\left(z-z_{i}\right)^{2}\left(e_{s}^{2}-1\right)+a_{s}^{2}\left(1-e_{s}^{2}\right)\left(1+e_{s}\right)}}{1+e_{s}}+y_{i}$, and the slope equation can be written as

$$
y_{t 0}^{\prime}=\mp \frac{\left(z-z_{i}\right)\left(e_{s}^{2}-1\right)}{\left(1+e_{s}\right) \sqrt{\left(z-z_{i}\right)^{2}\left(e_{s}^{2}-1\right)+a_{s}^{2}\left(1-e_{s}^{2}\right)\left(1+e_{s}\right)}} .
$$

The point of intersection $\left(z_{b 0}, y_{b 0}\right)$ of the path $y_{t 0}$ with boundary $y_{\text {trap }}$ is determined from the equation $\left(x-x_{i}\right)^{2}+\left(y_{t 0}(x)-y_{i}\right)^{2}=R_{\text {trap }}^{2}$ at the condition $\sqrt{\left(z_{b 0}-z_{b}\right)^{2}+\left(y_{b 0}-y_{b}\right)^{2}}=\min$, which defines the point nearest to the point $\left(z_{b}, y_{b}\right)$.

Finally, the rotation angle can be determined from Eq. (11)

$$
\psi=\operatorname{arctg}\left[\frac{-\left(z_{b 0}-z_{\mathrm{i}}\right)}{1+e_{s}} \sqrt{\frac{1-e_{s}}{a_{s}^{2}-\frac{\left(z_{b 0}-z_{\mathrm{i}}\right)^{2}}{1+e_{s}}}}\right]-\operatorname{arctg}\left[y_{k b}^{\prime}\left(x_{b}\right)\right] .
$$

To determine the ion path after leaving the potential well, the tangent line equation (10) and coordinates of exit point are used: $y_{k f}(z)=y_{f}+y_{t}^{\prime}\left(z_{f}\right)\left(z-z_{f}\right)$. 


\section{NUMERICAL RESULTS AND DISCUSSION}

The developed model was used to describe the trajectories of ions extracted from the plasma duct exit to the substrate. To determine the configurations of the negative space charge regions associated with the bottle or cusped magnetic traps, the magnetic field distributions were calculated for the discussed coil configurations by using the finite element method. Distributions shown in Figs. 7(a)-7(c) correspond to the $y-z$ plane (Fig. 1); those shown in Fig. 7(d) correspond to the $x-z$ plane. The distributions shown in Figs. 7(a)-7(d) correspond to $B_{g} \uparrow B_{m 2} \uparrow, B_{m 1} \uparrow B_{g} \uparrow B_{m 2} \uparrow$, and $B_{m 1} \downarrow B_{g} \uparrow B_{m 2} \uparrow$ configurations, respectively.

The negative space charge regions were approximated by the spherical areas which projections to the coordinate planes are shown in Figs. 7 and 8. The parameters of the regions were chosen to provide the best fit of the calculations to the experiment. To explain the ion flux focusing at the plasma duct axis for $B_{g} \uparrow B_{m 2} \uparrow$ and $B_{m 1} \uparrow B_{g} \uparrow B_{m 2} \uparrow$ configurations at the distance of $150 \mathrm{~mm}$ from the plasma duct exit, formation of a large negative space charge region near the plasma duct exit is considered. This region is designated as " $R_{1}$ " in Figs. 8(a)-8(d). This region is smaller for $B_{m 1} \downarrow B_{g} \uparrow B_{m 2} \uparrow$ configuration, this explains the presence of a large flux of ions which are not affected by the negative space charge and are deposited near the substrate edge.

Extraction of the ion jets shown in Fig. 3 suggests that the existence of the regions designated as " $R_{2}$ " (Figs. 8(a)$8(d))$. These regions are formed by the magnetic traps between the plasma duct exit and the additional coils. The magnetic cusp existing between the additional coils for the
$B_{m 1} \uparrow B_{g} \uparrow B_{m 2} \uparrow$ configuration (Fig. 7(b)) suggests the formation of region " $R_{3}$ " in Fig. 8(b).

To describe the ion jet directed toward the substrate edge between magnetic mirrors along $x$ axis (Fig. 3), formation of region " $R_{3}$ " is considered (Fig. 8(d)). Plasma electrons, drifting across the magnetic field from the magnetic mirrors above the coils 1 and 2, are trapped in the magnetic field lines between the mirrors (Figs. 8(c) and 8(d)), and form this region.

Thus, the centers of the regions, the radii $R_{\text {trapi }}$, and the ratio $K_{q n-i}$ were fit to describe the experimental results

- $R_{\text {trap } 1}=0.1 \mathrm{~m}, K_{q n-R 1}=2.0 \times 10^{-6}, R_{\text {trap } 2}=0.09 \mathrm{~m}, K_{q n-R 2}$ $=10^{-5}\left(B_{g} \uparrow B_{m 2} \uparrow\right.$, Fig. 8(a));

- $R_{\text {trap } 1}=0.1 \mathrm{~m}, K_{q n-R 1}=2.0 \times 10^{-6}, R_{\text {trap } 2}=0.08 \mathrm{~m} ; K_{q n-R 2}$ $=10^{-5}, \quad R_{\text {trap } 3}=0.052 \mathrm{~m} ; K_{q n-R 3}=10^{-5} \quad\left(B_{m 1} \uparrow B_{g} \uparrow B_{m 2} \uparrow\right.$, Fig. 8(b));

- $R_{\text {trap } 1}=0.05 \mathrm{~m}, \quad K_{q n-R 1}=6.0 \times 10^{-6}, \quad R_{\text {trap } 2}=0.09 \mathrm{~m}$; $K_{q n-R 2}=10^{-5}, R_{\text {trap } 3}=0.056 \mathrm{~m} ; K_{q n-R 3}=10^{-5}\left(B_{m 1} \downarrow B_{g} \uparrow\right.$ $B_{m 2} \uparrow$, Figs. 8(c) and 8(d)).

The initial ion flux is considered as being ejected from some point $\left(0,0, z_{0}\right)$ in the coordinate system shown in Fig. 1. The ion current distribution was assumed to be Gaussian for $B_{g} \uparrow$ configuration (Figs. 4(a), 4(e), and 4(k)), and the width of the distribution was approximated for $z \in(0.15 \ldots 0.325)$ by the equation $\Delta r(z)=10^{-3}(53+6.24[\exp (z / 0.13)+(z / 0.13)$ $-1])$, with $z=0$ corresponding to the plasma duct exit and $z$-axis directed toward the substrate. Coordinate $z_{0}$ was determined as a point of intersection of the tangent line to the curve $\Delta r(z)$ at point $z=0.15 \mathrm{~m}$ :
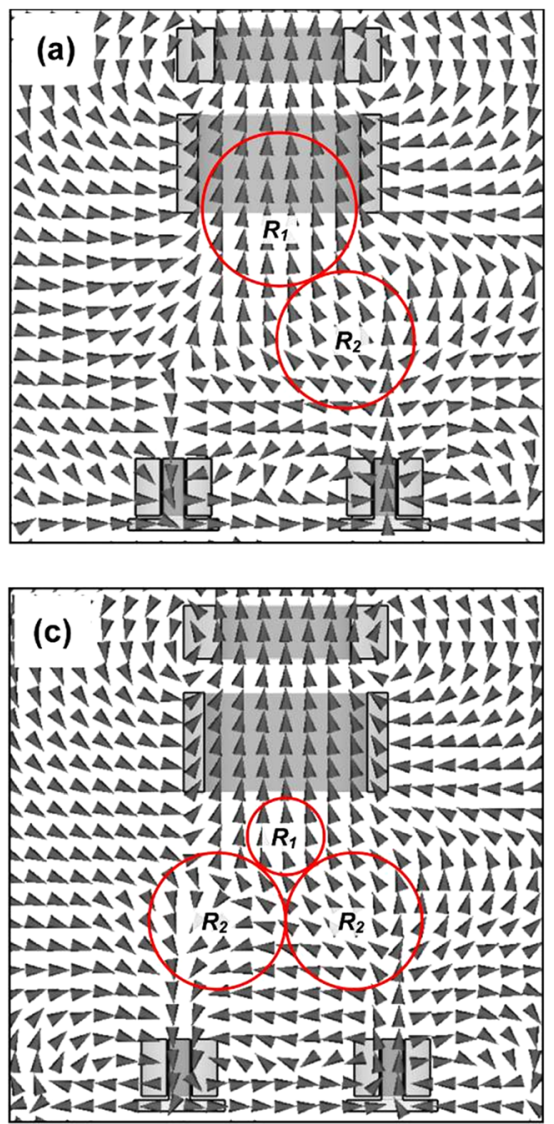
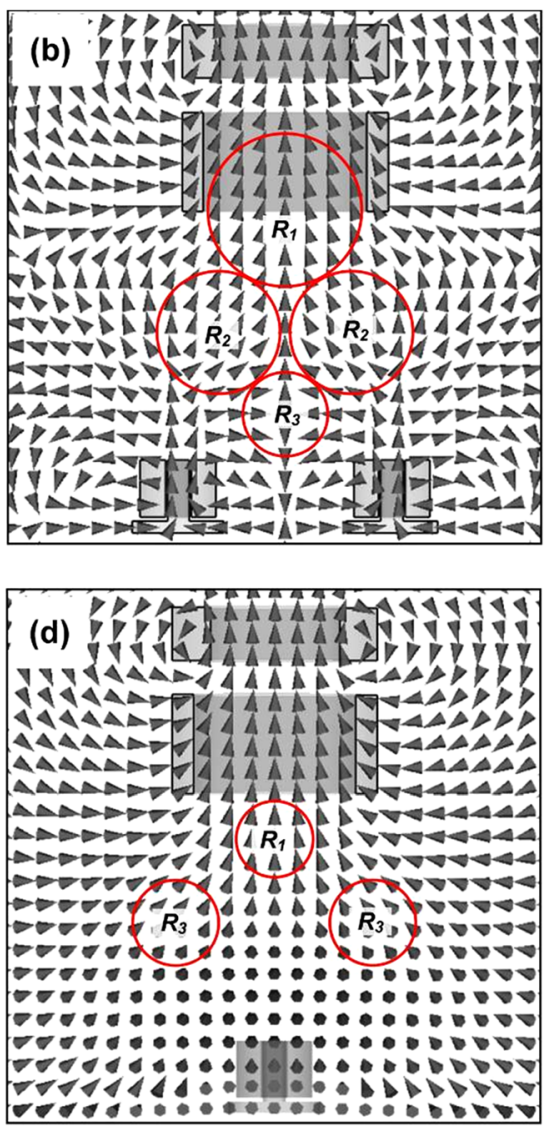

FIG. 7. Calculated magnetic field distributions and schemes of the magnetic traps: (a) $-B_{g} \uparrow B_{m 2} \uparrow$; (b) $-B_{m 1} \uparrow B_{g} \uparrow B_{m 2} \uparrow$; (c) $-B_{m l} \downarrow B_{g} \uparrow B_{m 2} \uparrow$ ( $y-z$ plane); and (d) $-B_{m 1} \downarrow B_{g} \uparrow B_{m 2} \uparrow(x-z$ plane). 

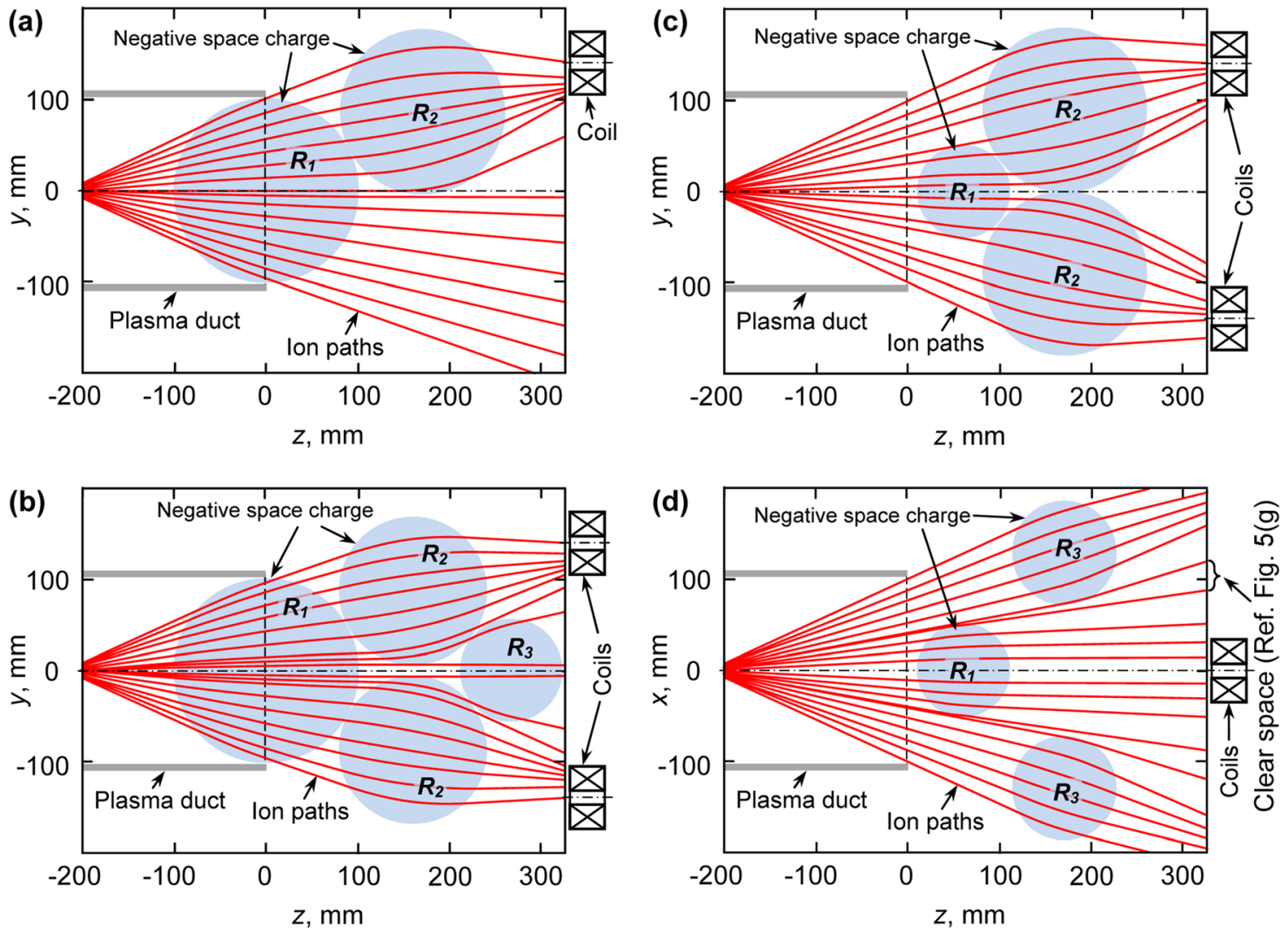

FIG. 8. Calculated ion paths for different configurations of the resulting magnetic field: (a) $-B_{g} \uparrow B_{m 2} \uparrow, I_{m 2}=4.0 \mathrm{~A}$; (b) $-B_{m 1} \uparrow B_{g} \uparrow B_{m 2} \uparrow, I_{m 1}=I_{m 2}=4.0 \mathrm{~A}$; (c) $-B_{m 1} \downarrow B_{g} \uparrow B_{m 2} \uparrow(y-z$ plane $), I_{m 1}=I_{m 2}=4.0 \mathrm{~A}$; and (d) $-B_{m 1} \downarrow B_{g} \uparrow B_{m 2} \uparrow(x-z$ plane $), I_{m 1}=I_{m 2}=4.0 \mathrm{~A}$.

$$
z_{0}=0.15-\frac{\Delta r(0.15)}{d \Delta r / d z(0.15)}=-0.216 \mathrm{~m}
$$

It should be noted that this value is close to the location of the front surface of the arc source cathode.

Then, the initial ion path was described by the straight line equation $y_{k b}(z)=k\left(z-z_{0}\right)$.

The ion paths were computed by using the model of Sec. IV, assuming that the plasma density is $n_{0}=10^{17} \mathrm{~m}^{-3}$, ion mass is $M=48 \times 1.67 \times 10^{-27} \mathrm{~kg}$ (titanium), and initial ion energy is $E_{\text {ion } 0}=50 \mathrm{eV} .^{48,49}$ The set of the calculated paths is shown in Fig. 8(a) for the $B_{g} \uparrow B_{m 2} \uparrow$ configuration, in Fig. 8(b) for the $B_{m 1} \uparrow B_{g} \uparrow B_{m 2} \uparrow$ configuration, and in Figs. 8(c) and 8(d) for the $B_{m 1} \downarrow B_{g} \uparrow B_{m 2} \uparrow$ configuration. It can be seen from these figures that the presence of the negative space charge explains the observed phenomena. Region $R_{1}$ affects the ion flux extracted from the arc source and focuses it slightly, but only a part of the ion flux is affected by region $R_{2}$ and directed toward the powered coil (Figs. 5(a) and 5(c)) for the $B_{g} \uparrow B_{m 2} \uparrow$ configuration (Fig. 8(a)). The symmetric operation of the coils in turn results in a similarly symmetric ion fluxes (Fig. 8(b)) with two ion jets directed towards the coils, see Fig. 5(e) for the $B_{m 1} \uparrow B_{g} \uparrow B_{m 2} \uparrow$ configuration.

The opposite powering of the coils results in the symmetric influence of the ion flux (Fig. 8(c) and 8(d)), confirming the results obtained by the probe measurements (Figs. 4(d), 4(h), and 4(n)). Extraction of the ion flux from the plasma source may result in the formation of the negative space charge region $R_{3}$ (Fig. $8(\mathrm{~d})$ ) on the way of the flux.
The relative position of the $R_{1}$ and $R_{3}$ regions explains the formation of a clear space between the thick and thin deposits observed in Fig. 5(g). At the same time, the opposite coil powering results in the smaller size of $R_{1}$ region (Figs. 8(c) and $8(\mathrm{~d})$ ) due to the presence of the magnetic cusp and the additional loss of the electrons to the chamber walls (Fig. 7(c)).

The electric field in the space charge regions was calculated by $E(r)=-(4 / 3) \pi e K_{q n} n_{0} K_{u} r: E(r)=-4760 r$ for $K_{q n}=2.0 \times 10^{-6}$, and $E(r)=-7930 r$ for $K_{q n}=10^{-5}$. The electric field can reach $720 \mathrm{Vm}^{-1}$ for the voltage drop of $32 \mathrm{~V}$ at the boundary of the largest region $R_{2}$ (Fig. $\left.7(\mathrm{c})\right)$. The excess of the electrons over the ions of about $K_{q n} \approx 10^{-5}$ generates a strong electric field. Therefore, the quasineutrality is significantly affected in magnetized plasmas, as compared to un-magnetized plasma with the excess of ions over the electrons of about $K_{q n} \approx 10^{-8.8}$. The calculations have demonstrated that the model adequately describes the observed phenomena and can be used to predict the variations of the ion current density distribution affected by the magnetic field.

\section{CONCLUSION}

This study shows that the magnetic field is a powerful tool to control ion fluxes extracted from the plasma. Electromagnetic coils matched with the guiding coil of the plasma source form an easily reconfigurable and adjustable control system where different configurations of the magnetic traps 
are produced. The plasma injected into this system interacts with the magnetic field, and the negative space charge regions are generated in the magnetic traps. These regions affect the plasma ions, thus affecting the ion current density distribution over the deposition substrate.

The proposed technique of generating a set of the magnetic traps for the plasma electrons allows controlling the ion current density over the particular area of the large substrate (about $400 \mathrm{~mm}$ dia.) in a range of $30-100 \%$, which can be a powerful tool in controlling the properties of the processed surface layer. The technique allows generation of both narrow and broad ion beams without changing the operation mode of the plasma source or the distance between the source and the substrate. The ion current density distribution can be varied from a Gaussian one to a complex multipeaked distribution, depending on the number and location of the magnetic coils. This allows sophisticated shaping of the ion flux into the time-averaged uniform distributions. This can be achieved by combining various numbers of the coils under substrate, their locations, scheme of powering, and relative motion of the substrate. Our results are closely relevant to various plasma processing technologies such as thin film deposition, surface layer modifications, and growth of nanostructures.

\section{ACKNOWLEDGMENTS}

This work was partially supported by the Australian Research Council, CSIRO's OCE Science Leadership Scheme, and Slovenian Research Agency.

${ }^{1}$ A. Anders, Handbook of Plasma Immersion Ion Implantation and Deposition (John Wiley \& Sons, New York, 2000).

${ }^{2}$ K. Ostrikov, U. Cvelbar, and A. B. Murphy, J. Phys. D: Appl. Phys. 44, 174001 (2011).

${ }^{3}$ K. Ostrikov, Plasma Nanoscience: Basic Concepts and Applications of Deterministic Nanofabrication (Wiley-VCH, Weinheim, Germany, 2008).

${ }^{4}$ X. Zhong, W. Zhou, X. Wu, L. Yuan, Q. Shu, W. Li, and Y. Xia, J. Phys. D: Appl. Phys. 40, 219 (2007).

${ }^{5}$ C. Chen and C. Liu, J. Electron. Mater. 34, 1408 (2005).

${ }^{6}$ S. Zhang, D. Sun, Y. Fu, and H. Du, Surf. Coat. Technol. 167, 113 (2003).

${ }^{7}$ F. Placido and D. Gibson, Chin. Optics Lett. 8, 49 (2010).

${ }^{8}$ F. F. Chen and J. P. Chang, Lecture Notes on Principles of Plasma Processing (Plenum/Kluwer, 2002).

${ }^{9}$ A. Anders, Thin Solid Films 518, 4087 (2010).

${ }^{10}$ I. Petrov, P. B. Barna, L. Hultman, J. E. Greene, J. Vac. Sci. Technol. A. 21, S117 (2003).

${ }^{11}$ R. Machunze, A. P. Ehiasarian, F. D. Tichelaar, and G. C. A. M. Janssen, Thin Solid Films 518, 1561 (2009).

${ }^{12}$ D. Manova, J. W. Gerlach, and S. Mandl, Materials 3, 4109 (2010).

${ }^{13}$ M. J. Grapperhaus, Z. Krivokapic, and M. J. Kushner, J. Appl. Phys. 83, 35 (1998).

${ }^{14}$ J. J. Cuomo, S. M. Rossnagel, and H. R. Kaufman, Handbook of Ion Beam Processing Technology (Noyes Publications, Park Ridge, NJ, USA, 1989).
${ }^{15}$ I. Levchenko and O. Baranov, Vacuum 72, 205 (2003).

${ }^{16}$ A. Anders, J. Phys. D: Appl. Phys. 40, 2272 (2007).

${ }^{17}$ O. Baranov, M. Romanov, S. Kumar, X. X. Zhong, and K. Ostrikov, J. Appl. Phys. 109, 063304 (2011).

${ }^{18}$ I. Levchenko, M. Romanov, and M. Keidar, J. Appl. Phys. 94, 1408 (2003).

${ }^{19}$ O. Baranov and M. Romanov, Plasma Processes Polym. 5, 256 (2008).

${ }^{20}$ O. Baranov and M. Romanov, Plasma Processes Polym. 6, 95 (2009).

${ }^{21}$ J. Hopwood, C. R. Guarnieri, S. J. Whitehair, and J. J. Cuomo, J. Vac. Sci. Technol. A 11, 147 (1993).

${ }^{22} \mathrm{~F}$. Chen, Introduction to Plasma Physics and Controlled Fusion (Plenum, New York, 1984).

${ }^{23}$ D. Tang and P. K. Chu, Appl. Phys. Lett. 82, 2014 (2003).

${ }^{24}$ Y. Kawai and Y. Ueda, J. Phys. 4, 235 (1997).

${ }^{25}$ T. Uchida, J. Vac. Sci. Technol. A 16, 1529 (1998).

${ }^{26}$ N. Savvides and B. Window, J. Vac. Sci. Technol. A 4, 196 (1986).

${ }^{27}$ I. Petrov, F. Adibi, J. E. Greene, W. D. Sproul, and W. D. Münz, J. Vac. Sci. Technol. A 10, 3283 (1992).

${ }^{28}$ J. Bohlmark, M. Östbye, M. Lattemann, H. Ljungcrantz, T. Rosell, and U. Helmersson, Thin Solid Films 515, 1928 (2006).

${ }^{29}$ A. Anders, Cathodic Arcs: From Fractal Spots to Energetic Condensation (Springer, New York, 2008).

${ }^{30}$ M. M. M. Bilek, A. Anders, and I. G. Brown, Plasma Sources Sci. Technol. 10, 606 (2001).

${ }^{31}$ O. Baranov, M. Romanov, and K. Ostrikov, Phys. Plasmas 16, 053505 (2009).

${ }^{32}$ Handbook of Vacuum Arc Science \& Technology: Fundamentals: Applications edited by R. L. Boxman, D. Sanders, and P. Martin (Noyes Publications, Park Ridge, NJ, 1995).

${ }^{33}$ M. Čerček, G. Filipič, T. Gyergyek, and J. Kovačič, Contrib. Plasma Phys. 50, 909 (2010).

${ }^{34}$ M. A. Lieberman and A. J. Lichtenberg, Principles of Plasma Discharges for Materials Processing (Wiley Interscience, New York, 1994).

${ }^{35}$ H. Nogami, Y. Ogahara, K. Mashimo, Y. Nakagawa, and T. Tsukada, Plasma Sources Sci. Technol. 5, 181 (1996).

${ }^{36}$ A. I. Morozov and V. V. Savelyev, in Reviews of Plasma Physics, edited by B. B. Kadomtsev and V. D. Shafranov (Consultant Bureau, New York, 2000), Vol. 21, p. 203.

${ }^{37}$ K. N. Ostrikov, M. Y. Yu, and N. A. Azarenkov, J. Appl. Phys. 84, 4176 (1998).

${ }^{38}$ N. A. Azarenkov, I. B. Denysenko, and K. N. Ostrikov, J. Phys. D.: Appl. Phys. 28, 2465 (1995).

${ }^{39}$ M. M. M. Bilek and I. G. Brown, Rev. Sci. Instrum. 69, 3353 (1998).

${ }^{40}$ R. C. Davidson, Phys. Fluids 19, 1189 (1976).

${ }^{41}$ D. B. Boercker, D. M. Sanders, J. Storer, and S. Falabella, J. Appl. Phys, 69, 115 (1991).

${ }^{42}$ R. D. White, R. E. Robson, S. Dujko, P. Nicoletopoulos, and B. Li, J. Phys. D: Appl. Phys. 42, 194001 (2009).

${ }^{43}$ X. Shi, Y. Q. Tu, H. S. Tan, and B. K. Tay, IEEE Trans. Plasma Sci. 24, 1309 (1996).

${ }^{44}$ B. Alterkop, E. Gidalevich, S. Goldsmith, and R. L. Boxman, J. Phys. D: Appl. Phys. 29, 3032 (1996).

${ }^{45}$ B. Alterkop, E. Gidalevich, S. Goldsmith, and R. L. Boxman, J. Phys. D: Appl. Phys. 41, 105211 (2008).

${ }^{46}$ I. I. Aksenov, A. N. Belokhvostikov, V. G. Padalka, N. S. Repalov, and V. M. Khoroshikh, Plasma Phys. Controlled Fusion 28, 761 (1986).

${ }^{47}$ A. Vesel and M. Mozetič, Vacuum 80, 253 (2005).

${ }^{48}$ A. Anders and G. Yu. Yushkov, J. Appl. Phys. 91, 4824 (2002).

${ }^{49}$ J. Kutzner and H. C. Miller, J. Phys. D: Appl. Phys. 25, 686 (1992).

${ }^{50}$ L. D. Landau and E. M. Lifshitz, Mechanics (Butterworth-Heinemann, 1976).

${ }^{51}$ E. M. Purcell, Electricity and Magnetism (Mcgraw-Hill, 1965). 